كتاب : "وسائل الإعلام الاجتماعية: مقدمة نقدية"

\title{
Social Media: A Critical Introduction
}

\section{تأليف : Christian Fuchs}

\section{$\underline{\text { (USA : Sage Publications, 2014) }}$}

إعداد: أ.د. شيماء ذوالفقار زغيب

يقدم كريستيان فوكس Christian Fuchs الأستاذ بجامعة وستمنستر Westminster University مقدمة نقدية" Social Media: A Critical Introduction في أحد عشر الابه

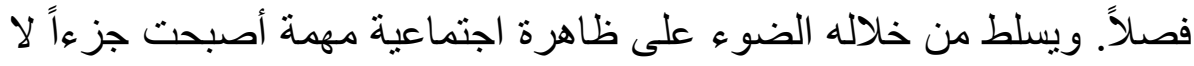

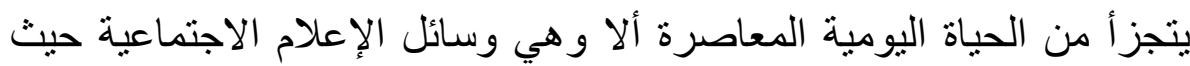

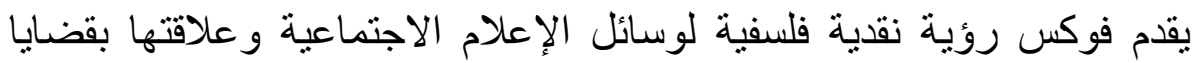

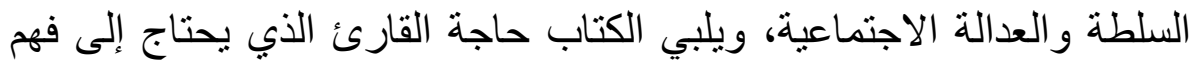

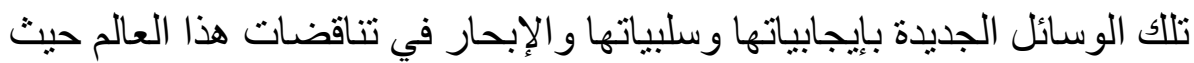

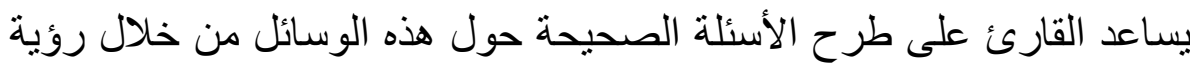
نقدية توضح علاقات التأثير و التأثر داخل هذا العالم المركب. ويشير فوكس إلى أن هدف الكتاب هو تحليل و اقع وسائل الإعلام الاجتماعية

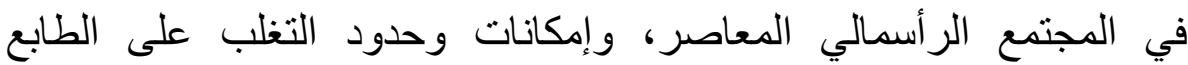
الر أسمالي المؤسس لهذه الوسائل. ويتناول فوكس وسائل الإعلام الاجتماعية العالية

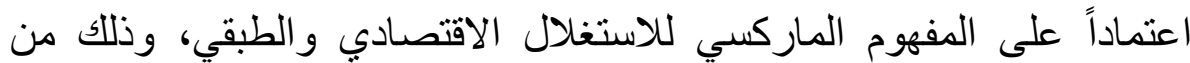
خلال التركيز على الاقتصاد السياسي لكل من فيسبوك وتوينر وجوجل الآنل

$$
\text { * أستاذ بقسم الإذاعة و التليفزيون - كلية الإعلام - جامعة القاهرة. }
$$


ويوتيوب، ويشير إلى أن تلك المواقع تعكس علاقات القوة في المجتمع

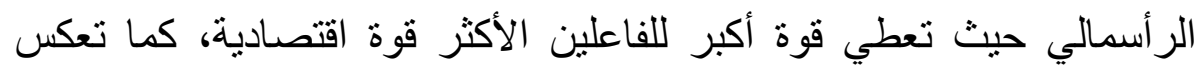

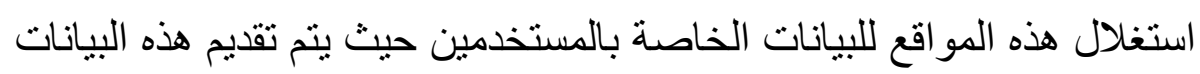

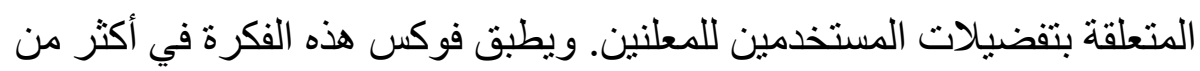

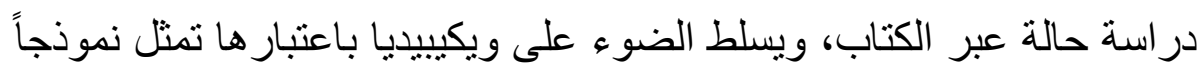

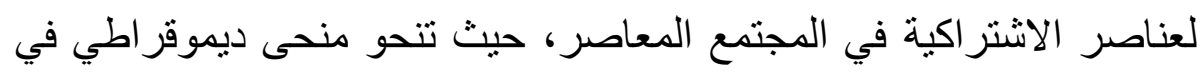

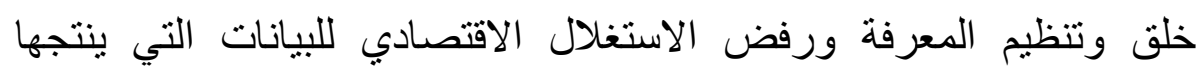

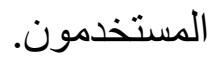

وقد جاء الفصل الأول كمقدمة لتوضيح ما يقصده المؤلف بالمقدمة النقدية

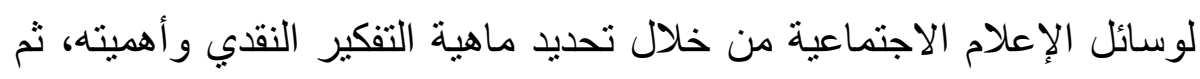

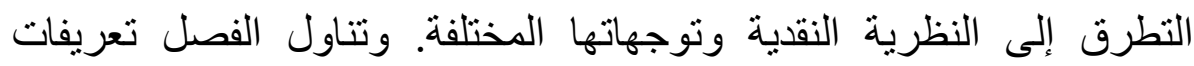

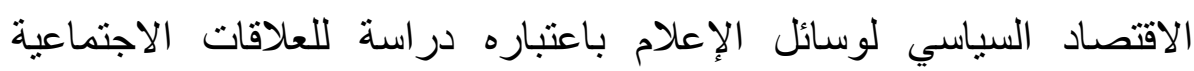

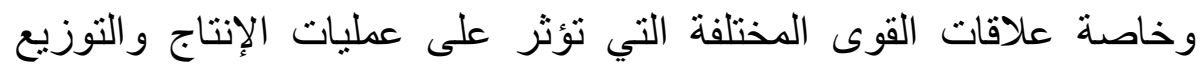

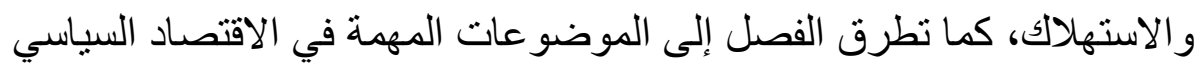

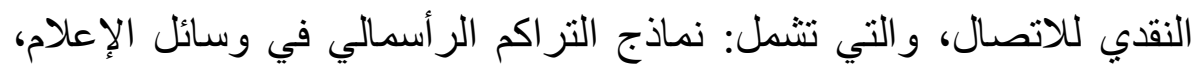

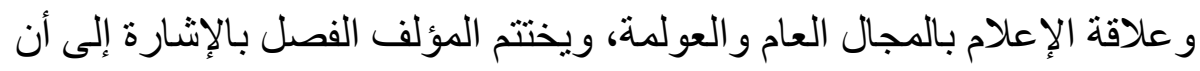

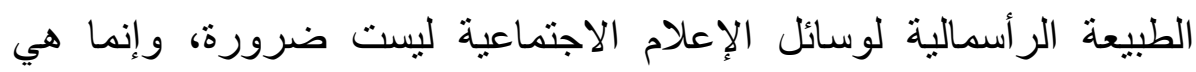

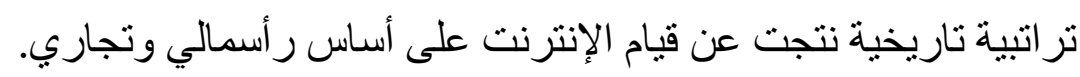

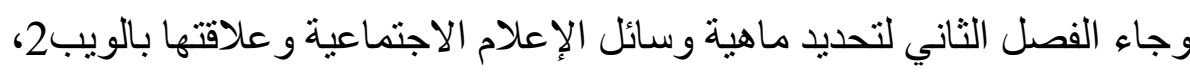

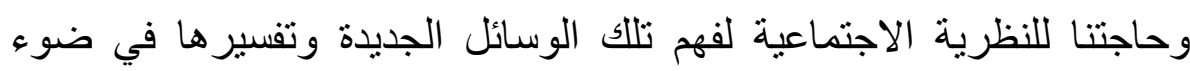

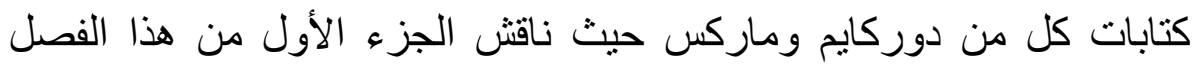

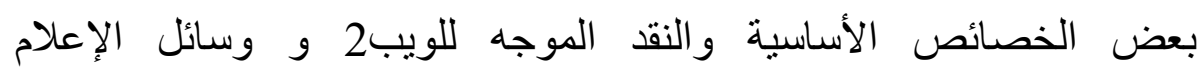

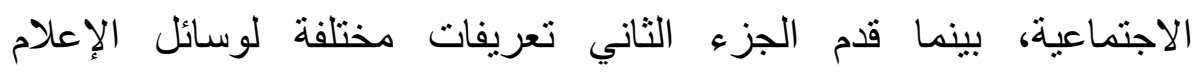


الاجتماعية، و اختتم المؤلف الفصل بإمكانية الاستفادة بما قدمه علماء الاجتماع في بناء نموذج يساعد في فهم تلك الوسائل.

وقدم الفصل الثالث علاقة وسائل الإعلام الاجتماعية بثقافة المشاركة و علاقتها بالسياسة وديموقراطية المشاركة، وقد بدأ الفصل بتعريف ثقافة المشاركة

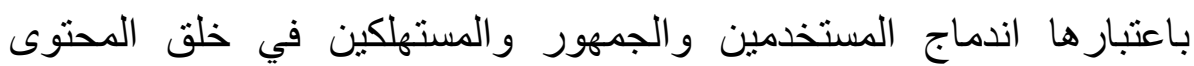
و الثقافة، ثم تناول الفصل الدور الذي يقوم به المستخدم غير مدفوع الأجر من أنشطة من شأنها خلق عائد مادي يستفيد به القائمون على المواقع المختلفة. ويستعرض الفصل الر ابع النظرية الاجتماعية في عصر المعلومات، كما يتناول فكرة الاتصال الجماهيري الذاتي، وقوة الاتصال في مجتمع الثبكات، من خلال ثورات الربيع العربي أو ما أطلق عليه ثورات تويتر وفيسبولك وحركة احتلوا M. Castells وول ستريت. ويقوم الفصل على منظور مانويل كاستيلز (أستاذ تكنولوجيا الاتصال والمجتمع في مدرسة أنينبرج للاتصال بجامعة ماتسن كاليفورنيا) ومفهومه عن قوة الاتصال في إطار وسائل الإعلام الاجتماعية فيما أطلق عليه المجتمع الثبكي Network Society حيث يعتبر الاتصال عبر فيرة

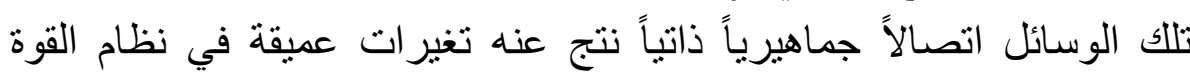
بالمجتمع، ويناقش فوكس عبر هذا الفصل رؤية كاستلز ويحللها إذ ير اها تفتقر لئر

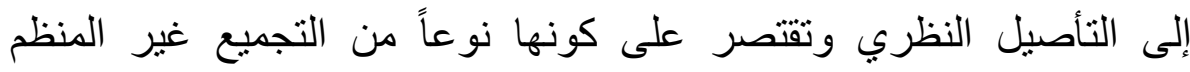
لمجموعة من الملاحظات، ويرى فوكس أن رؤية كاستلز لثورات الربيع Techno- العربي وحركة احتلوا وول ستريت تعبر عن النشوة التكنولوجية euphoria كاستلز أن هذه الثور ات و الحركات نبعت من عالم الإنترنت وتعيش وتثفاعل في الأساس من خلاله. 
ويتناول الفصل الرابع أيضاً تعريفات مختلفة لدفهوم القوة وفقاً للأبعاد المجتمعية المختلفة: الاقتصادية والسياسية و الثقافية، ويقام الفصل مقارنة بين

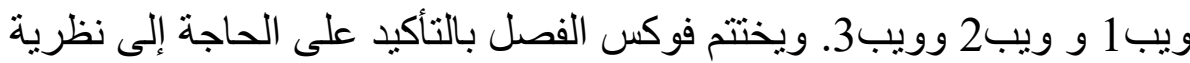

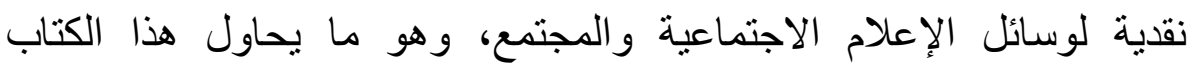
المساهمة في خلق بعض أسسها.

ويتناول الفصل الخامس الاقتصاد السياسي لوسائل الإعلام الاجتماعية من

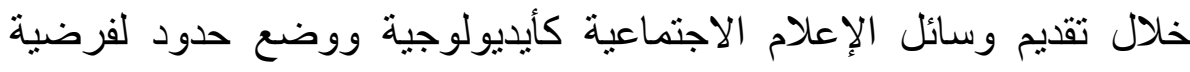

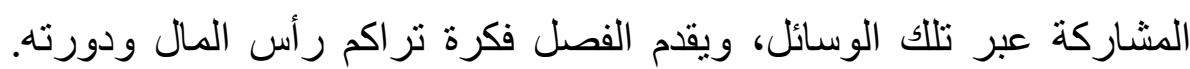
ويناقش فوكس من خلال الفصل حدود خاصية المشاركة في كل من يوتيوب

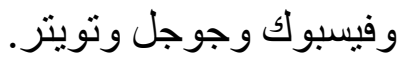

ويخلص المؤلف في نهاية ذلك الفصل إلى أن وسائل الإعلام الاجتماعية لا تقوم

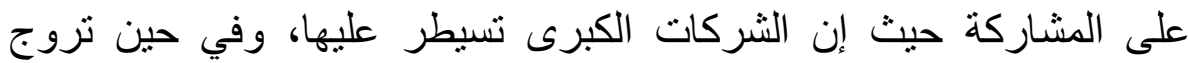

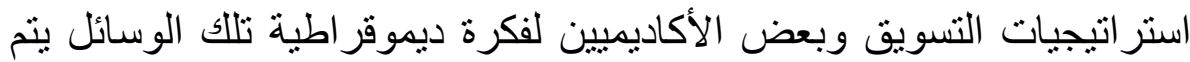
تجاهل مظاهر الرأسمالية التي تسيطر عليها، والتي تكرس فكرة استغلال تلاكل الثركات للأفر اد المستخدمين لتلالك الرسائل و استغلال ما ينتجو نه من خلالكها. ويستعرض الفصل الساد محرك البحث جوجل: إيجابياته وسلبياته، و الاقتصاد السياسي لهذا الدحرك والأيديولوجية التي يقوم عليها، كما يحلل الئل

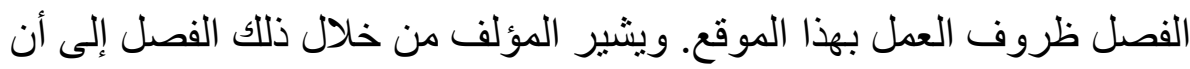

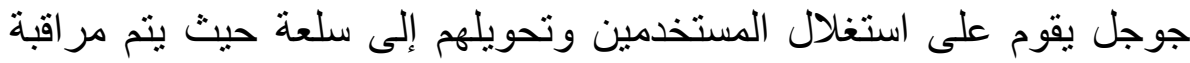

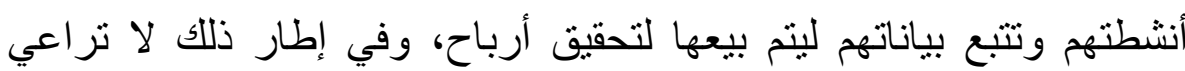
شركة جوجل قضايا الخصوصية. 
ويتناول الفصل السابع موقع التو اصل الاجتماعي فيسبوك من حيث مدى تهديده لخصوصية المستخدمين، والاقتصاد السياسي لهذا الموقع و الأيديولوجية التي يقوم عليها ومدى الاستخلال الذي قد يتم من خلاله، وبطرح بعض البدائل

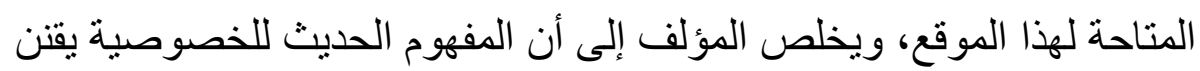

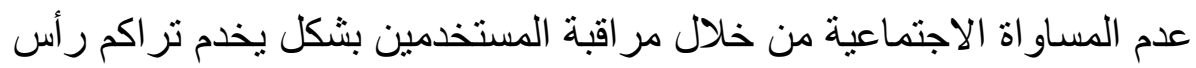
المال لدى القائمين على الموقع و المعلنين، وينتقد فوكس الدر اسات التي أجريت

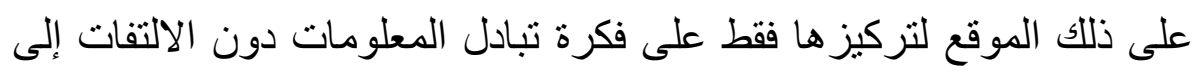
فكرة استغلال المستخدمين ودون تبني وجهة نظر نقدية للاقتصاد السياسي لهذا

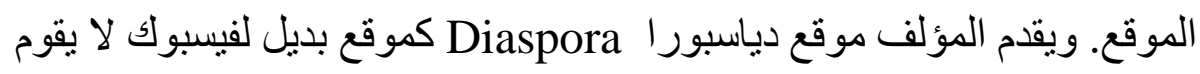

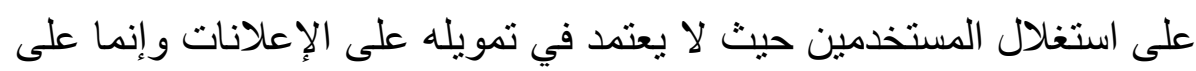
التبر عات، ويعتمد على الملكية الذاتية للبيانات.

ويستعرض الفصل الثامن موقع التواصل الاجتماعي تويتر وعاقلته بالديموقر اطية و الثور ات، ويجيب على التساؤل عما إذا كان تويتر مجالاً عاماً جديداً، ويعرض الفصل مفهوم هايبرماس عن المجال العام عبر تويتر والاقتصاد السياسي لهذا الموقع. ويخلص فوكس في نهاية الفصل إلى أن نوينر

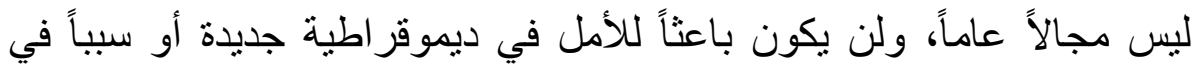

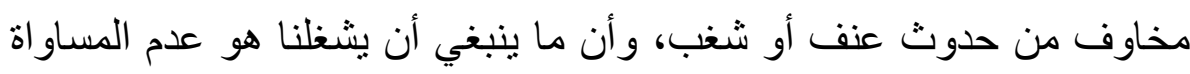
في المجتمع والتي تتعكس من خلال ذللك الموقع، وكيف يمكننا الحد منها، ويرى فوكس أن وسائل الإعلام الاجتماعية لا تحدث ثور فئ فات أو مظاهرات، ولكنها تحمل خصائص متناقضة في مجتمعات مليئة بالتناقضات.

ويتناول الفصل التاسع موقع ويكليكس محاولاً الإجابة عن إمكانية جعل القوة تتسم بالثفافية وعلاقة ويكليكس بالليبر الية والاشتر اكية، و علاقته بالصحافة ولته

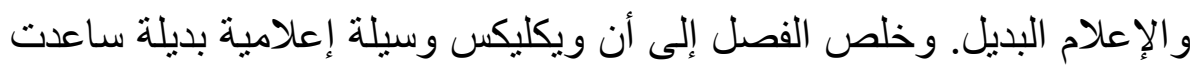


على جعل هياكل السلطة أكثر شفافية، وأعطت وزناً أكبر للسياسة، وتتسم بتحيز ليبر الي يركز على الحكم الرشيد، ولكن ينقصه فهم العلاقات الطبقية

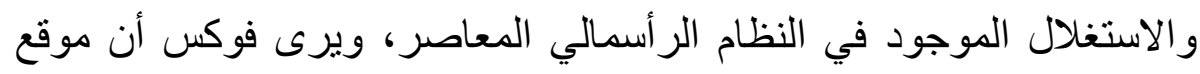
ويكليكس لديه الإمكانات التي تؤهله ليكون وسيلة مراقبة اشتر اكية، ولكن

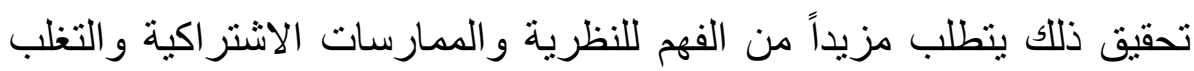
على تحيزه الليبر الي.

ويقدم الفصل العاثر ويكيبيديا كثكل ديموقراطي جديد من العمل والإنتاج

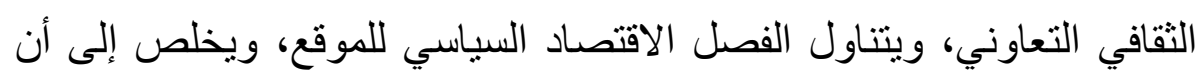

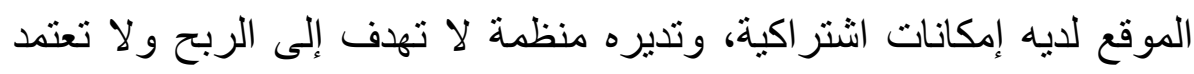

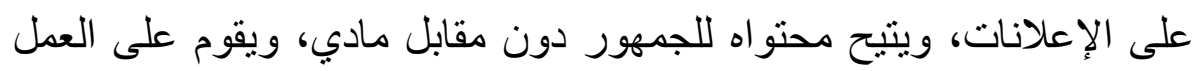
التطو عي.

ويختم فوكس الكتاب بتقديم خلاصة في الفصل الحادي عشر عن المفهوم الحقيقي لوسائل الإعلام الاجتماعية وبدائلها والأبديولوجيات التي تقوم عليها، وكيفية مقاومة الاستخلال الذي قد يتم بو اسطتها من خلال قو انين حماية البيانات و السياسات الإعلانية المختلفة.

وفي حين يقدم فوكس في كتابه فهماً دقيقاً للاقتصاد السياسي لوسائل الإعلام

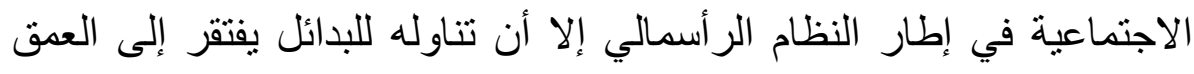
النظري والإمبريقي، حيث يقتصر في تناوله الإمبريقي للبدائل على ويكيبيديا،

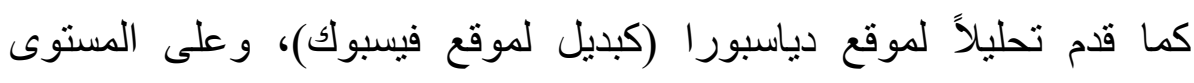

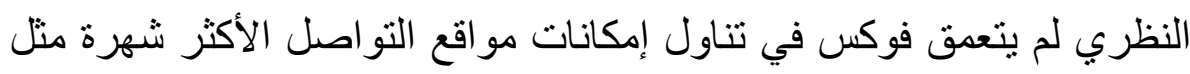

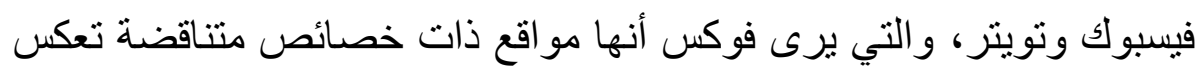
صر اعات طبقية بين مجمو عات مسيطرة ومجمو عات خاضعة. 
وفي المجمل فإن كتاب "وسائل الإعلام الاجتماعية: مقدمة نقدية" كتاب مهم لا غنى عنه للمهتمين و الدارسين بمجال الإعلام الجديد وتكنولوجيا الإنترنت. 